\title{
Joint T1 and Brain Fiber Diffeomorphic Registration Using the Demons
}

\author{
Viviana Siless ${ }^{1,2}$, Pamela Guevara ${ }^{2,3}$, Xavier Pennec ${ }^{4}$, and Pierre Fillard ${ }^{1,2}$ \\ 1 Parietal Team, INRIA Saclay-Île-de-France, Saclay, France \\ viviana.siless@inria.fr \\ http://parietal.saclay.inria.fr \\ 2 CEA, DSV, I²BM, Neurospin bât 145, 91191 Gif-Sur-Yvette, France \\ 3 University of Concepción, Concepción, Chile \\ 4 Asclepios Team, INRIA Sophia Antipolis Méditerraneé, Sophia Antipolis, France
}

\begin{abstract}
Non-linear image registration is one of the most challenging task in medical image analysis. In this work, we propose an extension of the well-established diffeomorphic Demons registration algorithm to take into account geometric constraints. Combining the deformation field induced by the image and the geometry, we define a mathematically sound framework to jointly register images and geometric descriptors such as fibers or sulcal lines. We demonstrate this framework by registering simultaneously $T_{1}$ images and 50 fiber bundles consistently extracted in 12 subjects. Results show the improvement of fibers alignment while maintaining, and sometimes improving image registration. Further comparisons with non-linear $T_{1}$ and tensor registration demonstrate the superiority of the Geometric Demons over their purely iconic counterparts.
\end{abstract}

Keywords: Registration, neural fibers, diffeomorphism, Demons Algorithm, intensity-base registration, tensor-base registration.

\section{Introduction}

Image registration is undoubtedly one of the most active area of research in medical imaging. Within inter-individual comparison, registration should align images as well as cortical and internal structures such as sulcal lines and fibers.

Non-linear registration algorithms can be categorized into three types: iconic, geometric and hybrid. Iconic, or image-based registration [1 2 23] consists in finding a voxel-wise mapping between a source and a target image. Schematically, iconic registration is mainly driven by the image contours (e.g., boundaries between white and grey matter). This approach suffers from the aperture problem: without prior knowledge, it is difficult to choose between two structures with similar contrast in the target image. Similarly, brain white matter, while being composed of neural fibers connecting cortical areas together, appears uniformly white in $T_{1}$ images, giving no relevant information to the iconic registratrion. Diffusion Tensor Imaging (DTI) can be used to reveal the microscopic structure of the white matter. Tensor-based registration was recently proposed to improve 
white matter alignment 4,5. However, misregistration may persist in regions where the tensor field appears uniform, as shown in [6].

Geometric registration specifically targets the alignment of Structures of Interest (SOI), such as in [7] for cortical surfaces, or [6] for fiber bundles. While those clearly improve SOI registration, they are in general not suitable for inferring a volumetric mapping between two subjects and cannot be used for comparing other structures than those used specifically during registration.

Hybrid techniques propose to jointly consider SOI and images during registration. For instance, 8 8] used the mathematical framework of the currents to simultaneously register images and geometric descriptors, while [10] proposed a Markovian solution to the same problem.

In this work, we propose to extend a well-established and efficient registration algorithm: the Demons. First introduced by Thirion et al. [1, and further extended by Vercauteren et al. [11 in a diffeomorphic version, the Demons have been widely used in the community for their efficiency and accuracy.

The rest of the paper is organised as follows. First, we propose a mathematically sound extension of the Demons, the Geometric Demons (GD), taking into account geometric constraints. Second, we evaluate the Geometric Demons with fiber constraints on a dataset of 12 subjects and compare them with their scalar and tensor versions [5], before concluding.

\section{The Geometric Demons}

\subsection{The Diffeomorphic Demons}

Image registration looks for a displacement field $s$ between a fixed $F$ and moving $M$ image, that maps as accurately as possible corresponding structures in both images. Finding $s$ is often treated as an optimization problem whose solution is defined by the minimum of the following energy: $E(s)=\frac{1}{\sigma_{i}^{2}} \operatorname{Sim}_{I}(F, M \circ s)+$ $\frac{1}{\sigma_{T}^{2}} \operatorname{Reg}(s)$, where $\operatorname{Sim}_{I}$ is a similarity measure between images and Reg a regularization term. The amount of regularization is controlled with $\sigma_{T}$ while $\sigma_{i}$ accounts for the image noise. In this work, the image similarity is defined as the sum of square differences $(\mathrm{SSD}): \operatorname{Sim}_{I}(F, M)=\|F-M\|^{2},\|\cdot\|$ being the $L_{2}$ norm. The regularization is chosen to be the harmonic energy: $\operatorname{Reg}(s)=\|\nabla s\|^{2}$.

In practice, this minimization is often intractable. To overcome this, Thirion et al. 1 introduced an auxiliary variable, the correspondence field $c$, to account for uncertainty of the displacement field. In other words, $c$ is an exact realization of point-to-point correspondences between images while $s$ allows for some errors. This leads to the following energy functional:

$$
E(c, s)=\frac{1}{\sigma_{i}^{2}} \operatorname{Sim}(F, M \circ c)+\frac{1}{\sigma_{x}^{2}} \operatorname{dist}(s, c)^{2}+\frac{1}{\sigma_{T}^{2}} \operatorname{Reg}(s),
$$

The term $\operatorname{dist}(s, c)^{2}$ imposes the displacement field $s$ to be close to the correspondence field $c$. $\sigma_{x}$ weights the spatial uncertainty on the deformation. 
The energy minimization is performed by alternating minimization w.r.t. $c$ and $s$. In [11], small deformations parametrized by a dense displacement field $u$ are used: $c$ is given by $s$ composed with the exponential map of $u$ (in the Lie group sense): $c \leftarrow s \circ \exp (u)$. Using the exponential map ensures the result to be diffeomorphic. The algorithm consists thus in the following steps:

1. Given $s$, find the optimal update field $u$ minimizing Eq. 1.

2. Let $c \leftarrow s \circ \exp (u)$

3. Minimize Eq. 1 by convolving $c$ with a Gaussian kernel $K_{\text {diff }}: s \leftarrow K_{\text {diff }} \star c$

4. Iterate until convergence.

Implementation details for each step is given in 11. In the next section, we show how to adapt the Demons framework to include geometric constraints.

\subsection{Adding Geometric Constraints to the Demons}

To add geometric constraints in the Demons framework, $c$ should ideally carry information from both image and geometry. Let us denote by $\mathcal{G}^{F}$ (resp. $\mathcal{G}^{M}$ ) the fixed (resp. moving) geometric descriptors. We propose the following formulation:

$$
\begin{aligned}
E(c, s)= & \frac{1}{\sigma_{i}^{2}}\left[\operatorname{Sim}_{I}(F, M \circ c)+\operatorname{Sim}_{G}\left(c \star \mathcal{G}^{F}, \mathcal{G}^{M}\right)\right]+ \\
& \frac{1}{\sigma_{x}^{2}} \operatorname{dist}(s, c)^{2}+\frac{1}{\sigma_{T}} \operatorname{Reg}(s),
\end{aligned}
$$

where $\operatorname{Sim}_{I}$ is the image similarity measure, $\operatorname{Sim}_{G}$ the geometry similarity measure, and $c \star \mathcal{G}^{F}$ denotes the action of $c$ on the geometry.

Following [11, we parametrize $c$ by an update field, which in this case will be the additive combination of an image update field $u_{I}$ and a geometric update field $u_{G}$. Ideally, one should use $u_{G}$ only where geometric information is relevant and use $u_{I}$ elsewhere. Thus, if we denote by $\Omega_{G}$ the definition domain of $u_{G}$ (regions where geometric correspondences are present), the definition domain of $u_{I}$ is $\Omega_{I}=\Omega-\Omega_{G}$, of which is equivalent to having non-intersecting domains, the union covers the entire domain $\Omega: \Omega_{I} \cap \Omega_{P}=\emptyset, \Omega_{I} \cup \Omega_{G}=\Omega$. Under this assumption, $c$ can be seen as the combination of both update fields: $c=$ $\exp \left(u_{I}+u_{G}\right)$. We can also say that $c \star \mathcal{G}^{F}=\exp \left(u_{G}\right) \star \mathcal{G}^{F}$ and $M \circ c=M \circ \exp \left(u_{I}\right)$.

Furthermore, since $u_{I}$ and $u_{G}$ have distinct domains, we can write: $\operatorname{dist}(s, c)^{2}=$ $\int_{\Omega_{G}}\left\|u_{G}\right\|^{2}+\int_{\Omega_{I}}\left\|u_{I}\right\|^{2}$. Optimizing Eq. 2 w.r.t. to $u_{I}$ leads to the minimization of $E_{I}\left(s, u_{I}\right)=\frac{1}{\sigma_{i}^{2}} \operatorname{Sim}_{I}\left(F, M \circ s \circ \exp \left(u_{I}\right)\right)+\frac{1}{\sigma_{x}^{2}} \int_{\Omega_{I}}\left\|u_{I}\right\|^{2}$, which is the same formulation as the diffeomorphic Demons. Optimizing Eq. 2 w.r.t. to $u_{G}$ leads to minimizing the following energy:

$$
E_{G}\left(s, u_{G}\right)=\frac{1}{\sigma_{i}^{2}} \operatorname{Sim}_{G}\left(s \circ \exp \left(u_{G}\right) \star \mathcal{G}^{F}, \mathcal{G}^{M}\right)+\frac{1}{\sigma_{x}^{2}} \int_{\Omega_{G}}\left\|u_{G}\right\|^{2},
$$

Finally, the Geometric Demons algorithm can be formulated as such:

1. Given $s, u_{G}$, compute the update field $u_{I}$ as for the diffeomorphic Demons

2. Given $s, u_{I}$, compute the update field $u_{G}$ by minimizing Eq. 3. 
3. Let $c \leftarrow s \circ \exp \left(u_{I}+u_{G}\right)$

4. Given $c$, let $s \leftarrow K_{\text {diff }} \star c$

5. Iterate until convergence

Steps 1, 3 and 4 are similar to the diffeomorphic Demons. Note that step 4 ensures that the combined correspondence field is smooth. In the following, we detail the computation of $u_{G}$ in the case of point sets as geometric descriptors.

Calculation of $\boldsymbol{u}_{\boldsymbol{G}}$ for Point Sets. There are numerous ways to measure similarity between geometrical primitives such as with the Hausdorff distance, the Closest Point distance (CPD), or even more sophisticatedly, with the currents. Our aim is to jointly minimize image and geometric energies within the Demons's framework, thus we focus on the overall algorithm behaviour using a simple metric for now.

Let us consider our geometric descriptors as point sets: $\mathcal{G}=\left\{x_{i}\right\}_{i \in[1, N]}$, being $N$ the number of points. Let us denote by $\pi_{i}$ the point index in $\mathcal{G}^{M}$ closest to point $i$ in $\mathcal{G}^{F}$. We define the similarity measure between point set with the CPD:

$$
\operatorname{Sim}_{G}\left(\mathcal{G}^{F}, \mathcal{G}^{M}\right)=\frac{1}{N} \sum_{i=1}^{N}\left\|x_{i}^{F}-x_{\pi_{i}}^{M}\right\|_{2}^{2}
$$

Let us define the action of the correspondence field $c$ on a point set as: $c \star \mathcal{G}=$ $\left\{s \circ \exp \left(u_{G}\right)\left(x_{i}\right)\right\}_{i \in[1, N]} \approx\left\{s\left(x_{i}\right)+u_{G}\left(x_{i}\right)\right\}_{i \in[1, N]}$. Since we are dealing with sets of points, we choose to parametrize the dense update field $u_{G}$ by a finite set of vectors $u_{G, i}$ using radial basis function interpolation: $u_{G}(x)=\sum_{i=1}^{N} h(\| x-$ $\left.x_{i} \|\right) \lambda_{i} . h($.$\left.) is a radial function (we use h(x)=\|x\|\right) . \lambda_{i}$ are calculated such that $u_{G}\left(x_{i}\right)=u_{G, i} \forall i$. Let us define the matrix $A$ such that $[A]_{i, j}=h\left(\left\|x_{i}-x_{j}\right\|\right)$ $\left([A]_{i, j}\right.$ denotes the $i^{\text {th }}$ row and $j^{t h}$ column of $\left.A\right), \Lambda=\left[\lambda_{1}, \ldots, \lambda_{N}\right]$ the vector of $\lambda \mathrm{s}, H(x)$ the vector such that $[H(x)]_{i}=h\left(\left\|x-x_{i}\right\|\right)$ and $U=\left[u_{G, 1}, \ldots, u_{G, N}\right]$. We can write: $u_{G}(x)=H(x) A^{-1} U$. Solving $\nabla E_{G}\left(s, u_{G}\right)=0$ w.r.t. $u_{G}$ narrows down to optimization for the $u_{G, i}, \forall i$. After differentiation, we obtain:

$$
u_{G, i}=\frac{x_{\pi_{i}}^{M}-s\left(x_{i}^{F}\right)}{1+\frac{N \sigma_{i}^{2}}{\sigma_{x}^{2}}\left[H\left(s\left(x_{i}^{F}\right)\right) A^{-1}\right]_{i}}
$$

Defining $\Omega_{G}$ for Point sets. Since we want points to influence the deformation near the definition domain, we define the domain as the union of $\gamma$-radius balls $B$ centered at each coordinate $x_{i}$. We control the influence by varying $\gamma$ and thus, dilating the domain. We define a binary map $\Omega_{G}^{\gamma}=\bigcup_{i=1}^{N} B\left(x_{i}, \gamma\right)$. The domain of the image correspondence field is the complementary of $\Omega_{G}^{\gamma}: \Omega_{I}^{\gamma}=\Omega \backslash \Omega_{G}^{\gamma}$.

\section{Joint $T_{1}$ MRI and Brain Fiber Registration}

We now apply the GD the joint registration of $T_{1} \mathrm{MRI}$ and brain fibers. 


\subsection{Data Description}

Analysis was performed for 12 subjects of the NMR public database [12]. This database provides high quality T1-weighted images and diffusion data acquired with a GE Healthcare Signa 1.5 Tesla Excite II scanner. The diffusion data presents a high angular resolution (HARDI) based on 200 directions and a bvalue of $3000 \mathrm{~s} / \mathrm{mm} 2$ (voxel size of $1.875 \times 1.875 \times 2 \mathrm{~mm}$ ). Distorsion correction and fiber tractography and clustering were performed using the Brainvisa software package (http://brainvisa.info). Using [13], we obtained corresponding fiber bundles between several subjects and a single representative fiber for each bundle. About 100 bundles were consistently identified in all subjects. The 50 longest (25 in each hemisphere) were retained for the experiments. For each subject we apply affine registration from $B_{0}$ to $T_{1}$ and use the resulting transformation to align budles with $T_{1}$ images. Bundles were further simplified into point sets, which allows us to use the methodology presented in Sec. 2,

\subsection{Experiments}

Two experiments were conducted. First, we performed an exhaustive analysis of the parameter $\gamma$ of Sec. 2.2 to understand its effect on registration accuracy. Second, we compared the performance between the GD, the Scalar Demons (SD) and the Tensor Demons (TD). For both experiments, 11 subjects were registered onto one, arbitrary chosen as the target. Note that the deformation field obtained is the resampling deformation: it goes from target to source. As our deformation field is diffeomorphic we can invert it to display registered fibers onto the target.

Influence of $\gamma$. We varied $\gamma$ from 0 (no fiber influence, which is equivalent to $\mathrm{SD})$ to 3.0. Registration results for three values of $\gamma$ are shown in Fig. 11 Values of the image and fiber similarity measures for increasing values of $\gamma$ are reported in Fig. 1 (d) and (e). As expected, when $\gamma$ increases, fiber matching improves at the expense of image alignment. Indeed, when fibers have a large influence on their neighborhood, image-driven forces are discarded, leading to poor image registration. However, we noticed that a $\gamma$ value of 1.5 largely improves fiber alignment while keeping a good match between images. Notably, in some cases image matching is improved when using fiber as constraints compared to not using them at all, pointing out the fact that geometry may indeed help image registration to avoid local minima. In the sequel, a $\gamma$ of 1.5 will be used.

Comparison with Scalar and Tensor Demons. For SD, we registered all 11 $T_{1}$ images onto the target and applied the inverted deformation field to the bundles. For TD, we extracted tensors using 14 and registered them onto the target tensor image. Then, inverted deformation fields were applied to each subject's fibers in the DWI space. Finally, the linear transformation calculated between the target $B_{0}$ and $T_{1}$ images was applied to fibers to carry them to the $T_{1}$ space. We evaluated the fiber similarity measure between registered source and target fibers. Results for each method and each subject are reported in Fig. 2 . 


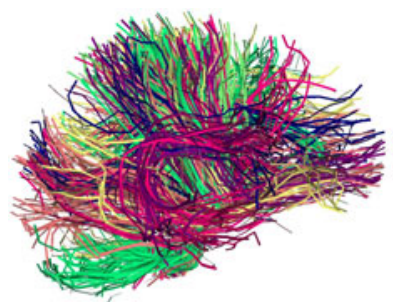

(a) $\gamma=0$

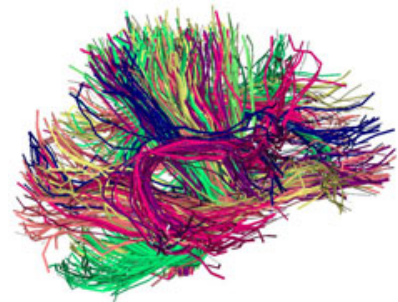

(b) $\gamma=1.5$

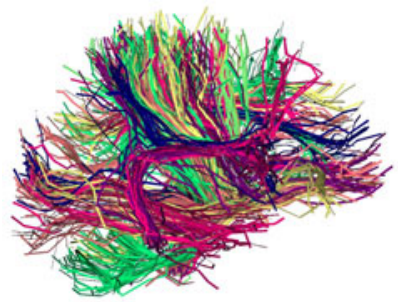

(c) $\gamma=3$

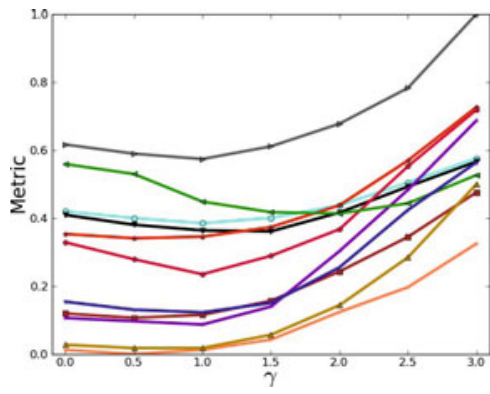

(d)

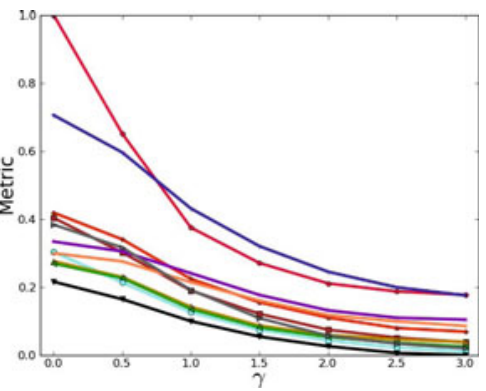

(e)

Fig. 1. Influence of $\gamma$ on the registration accuracy. Top: Fibers of 11 subjects were overlapped after registration with the Geometric Demons for three values of $\gamma$. Corresponding fibers in different subjects share colors. Bottom: Evolution of the image and fiber similarity measures with varying $\gamma$ is shown in (d) and (e) (one curve per subject). For each metric, values were scaled using min-max normalization.

As expected, TD improved fiber registration compared to SD. Similarly, GD further improved fiber alignment consistently for all subjects. However, the same set of fibers used for registration was used for performance evaluation. This favors our method as we explicitly optimize a metric evaluated on those fibers. For a fair evaluation, we measured in another experiment the fiber similarity on the 50 bundles that were left aside (100 bundles were extracted and only 50 were kept). In other words, we perform registration on half of the bundles and evaluate the result quality on the other half. Results are shown in Fig. 2 (e). We noticed a similar performance between TD and GD, both improving results obtained by SD. However, GD was only using sparse information from tensors over the set of fibers not being tested: having similar results as TD is thus very promising.

\section{Discussion}

As expected, GD increased fiber matching compared to the scalar and tensor versions while preserving a good match between images. Interestingly, the algorithm performed even better on images themselves in some subjects. Furthermore, when evaluating algorithm performance on a different set of fibers than those used for registration, we found that GD better registers missing structures 


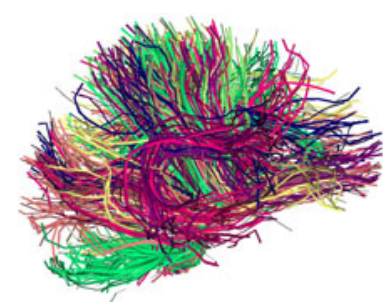

(a) Scalar Demons

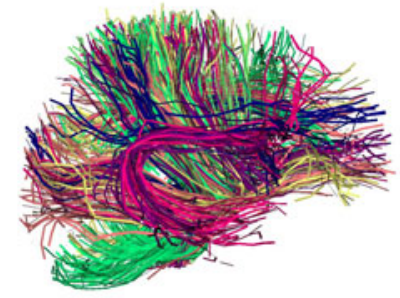

(b) Tensor Demons

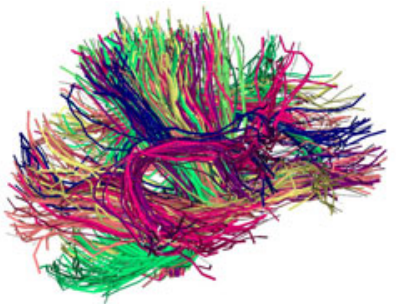

(c) Geometric Demons

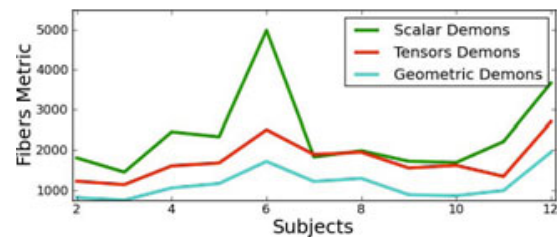

(d)

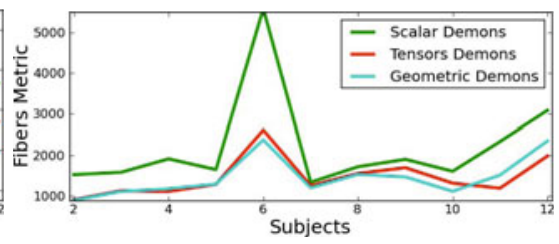

(e)

Fig. 2. Comparison of Scalar, Tensor and Geometric Demons. Top: Fibers of 11 subjects were overlapped after registering with: (a) Scalar Demons, (b) Tensor Demons, (c) Geometric Demons. Corresponding fibers in subjects share colors. Bottom: Fiber similarity metric for each subject and each method evaluated on the fibers set (d) used during registration and (e) left aside.

than SD, and performs similarly to TD. It also shows that a small set of fibers might be sufficient for a proper registration of the white matter across subjects.

By using labeled fibers instead of purely tensor information, we add relevant features that were previously extracted as prior such as region connection or fibers differently classified which should not be merged. Even though the efficacy of trusting fibers is open to discussion, classification of fibers is an active topic in research and we believe this information should not be discarded.

Nevertheless, application of GD to joint $T_{1}$ /fiber registration can be improved. First, the CPD imposes to have a fiber-to-fiber correspondence. Using [13] we consistently extract corresponding single fiber representatives from subjects, which is not optimal. Indeed, not all bundles can be reduced to a single line. We are working towards using the currents as in [6, which would allow us to directly use sets of lines instead of points. Second, the resampling deformation field had to be inverted to obtain the geometric deformation. A better strategy would be to use the log-domain diffeomorphic Demons [15] which optimizes the $\operatorname{logarithm}$ (in the Lie group sense) of the deformation field $s: l=\log (s)$. Then, the inverse field is easily obtained by taking the exponential of the opposite of $l$.

\section{Conclusion}

In this work, we presented an extension of the well-established Demons algorithm for non-linear registration taking into account geometric constraints. 
The framework is generic in the sense that any type of geometric descriptors, such as line or surfaces, can be incorporated given a differentiable similarity measure.

Within $T_{1}$ /fiber registration, the GD showed to perform better than their scalar and tensor versions: the obtained deformation field correctly aligns the $T_{1}$ images, and also better aligns fibers. This can be used to perform group studies targeting, at the same time, voxel-based morphometry (VBM) and shape analysis of structures of interest: a unique deformation field mapping simultaneously images and structures can be obtained. This gives a consistent framework for analyzing and comparing results between VBM and shape analysis.

As future work we plan to improve the similarity measure between geometric structures, i.e. using currents, and incorporate new structures such as sulcal lines or cortical surfaces. Another application is the combined registration and clustering of fibers: increased fiber registration can help clustering algorithms, which can in turn guide the registration.

Acknowledgements. This work was supported by the ANR (Agence Nationale de la Recherche) "programme blanc" KaraMetria number ANR-09-BLAN-033201 .

\section{References}

1. Thirion, J.P.: Image matching as a diffusion process: an analogy with Maxwell's demons. Medical Image Analysis 2(3), 243-260 (1998)

2. Beg, M.F., et al.: Computing large deformation metric mappings via geodesic flows of diffeomorphisms. IJCV 61(2), 139-157 (2005)

3. Rueckert, D., Aljabar, P., Heckemann, R.A., Hajnal, J.V., Hammers, A.: Diffeomorphic registration using B-splines. In: Larsen, R., Nielsen, M., Sporring, J. (eds.) MICCAI 2006. LNCS, vol. 4191, pp. 702-709. Springer, Heidelberg (2006)

4. Zhang, H., et al.: Deformable registration of diffusion tensor mr images with explicit orientation optimization. Medical Image Analysis 10(5), 764-785 (2006)

5. Yeo, B., et al.: Dt-refind: Diffusion tensor registration with exact finite-strain differential. IEEE Trans. Med. Imaging 28(12), 1914-1928 (2009)

6. Durrleman, S., et al.: Registration, atlas estimation and variability analysis of white matter fiber bundles modeled as currents. NeuroImage 55(3), 1073-1090 (2011)

7. Yeo, B., et al.: Spherical demons: Fast diffeomorphic landmark-free surface registration. IEEE Trans. Med. Imaging 29(3), 650-668 (2010)

8. Auzias, G., et al.: Diffeomorphic brain registration under exhaustive sulcal constraints. IEEE Trans. Med. Imaging (January 2011)

9. Ha, L.K., et al.: Image registration driven by combined probabilistic and geometric descriptors. In: Jiang, T., Navab, N., Pluim, J.P.W., Viergever, M.A. (eds.) MICCAI 2010. LNCS, vol. 6362, pp. 602-609. Springer, Heidelberg (2010)

10. Sotiras, A., et al.: Simultaneous geometric - iconic registration. In: Jiang, T., Navab, N., Pluim, J.P.W., Viergever, M.A. (eds.) MICCAI 2010. LNCS, vol. 6362, pp. 676683. Springer, Heidelberg (2010)

11. Vercauteren, T., et al.: Diffeomorphic demons: Efficient non-parametric image registration. NeuroImage 45(sup.1), S61-S72 (2009) 
12. Poupon, C., et al.: A database dedicated to anatomo-functional study of human brain connectivity. In: 12th HBM Neuroimage, Florence, Italie, vol. (646) (2006)

13. Guevara, P., et al.: Robust clustering of massive tractography datasets. Neuroimage 54(3), 1975-1993 (2011)

14. Fillard, P., et al.: Clinical DT-MRI estimation, smoothing and fiber tracking with log-Euclidean metrics. IEEE Trans. Med. Imaging 26(11), 1472-1482 (2007)

15. Vercauteren, T., et al.: Symmetric log-domain diffeomorphic registration: A demons-based approach. In: Metaxas, D., Axel, L., Fichtinger, G., Székely, G. (eds.) MICCAI 2008, Part I. LNCS, vol. 5241, pp. 754-761. Springer, Heidelberg (2008) 